\title{
SUMS OF STATIONARY RANDOM VARIABLES ${ }^{1}$
}

ENDERS A. ROBINSON

A sequence $x(t)(-\infty<t<\infty, t$ an integer) of elements in Hilbert space is called stationary if the inner product $(x(t+s), x(t))$ does not depend upon $t$. If the Hilbert space is $L^{2}$ space with probability measure, then $x(t)$ is a random variable and the sequence $x(t)(-\infty<t<\infty)$ is called a second-order stationary random process. Let $X$ be the closed linear manifold spanned by all the elements of the stationary process. Then Kolmogorov [1] has shown that the equation $x(t) U$ $=x(t+1),-\infty<t<\infty$, uniquely determines the unitary operator $U$ with domain and range $X$. Using the von Neumann [2] spectral representation of $U$, we obtain the spectral representation of the random process

$$
x(t)=\int_{-.5}^{.5} e^{2 \pi i u t} d x(0) E(u), \quad-\infty<t<\infty .
$$

The von Neumann [3] ergodic theorem, in the framework of Khintchine [4], is applicable, and shows that the average $\sum_{1}^{n} x(t) / n$ converges in the mean to the random variable $x(0)[E(0+)-E(0-)]$ as $n \rightarrow \infty$. In this paper we consider sums instead of averages; that is, we consider $\sum_{1}^{n} x(t)$, and establish the following theorem.

THEOREM. Let the random variables $x(t)(-\infty<t<\infty, t$ an integer $)$ be a second-order stationary random process with spectral distribution function $F(u)$. For variance $\left\{\sum_{1}^{n} x(t)\right\}$ to be bounded for all positive integers $n$, each of the following two conditions is necessary and sufficient:

$$
\int_{-.5}^{.5} \sin ^{-2} \pi u d F(u)<\infty .
$$

(2) There is a second-order stationary random process

$$
y(t)(-\infty<t<\infty) \text { satisfying } y(t)-y(t+1)=x(t) .
$$

Proof. (Necessary CONditions). We are given that variance $\left\{\sum_{1}^{n} x(t)\right\}<B$ for all positive integers $n$. Without loss of generality we assume that the $x(t)$ are centered so that their mean values are zero. Then

Presented to the Society, February 28, 1959; received by the editors April 6, 1959.

1 This paper was sponsored by the United States Army under Contract No. DA-11-022-ORD-2059 at the Mathematics Research Center, United States Army, Madison, Wisconsin. 


$$
\text { variance }\left\{\sum_{1}^{n} x(t)\right\}=\left(\sum_{1}^{n} x(t), \sum_{1}^{n} x(t)\right) \text {. }
$$

From the spectral representation we have

$$
\sum_{1}^{n} x(t)=\int_{-.5}^{.5} e^{2 \pi i u} \frac{1-e^{2 \pi i u n}}{1-e^{2 \pi i u}} d x(0) E(u)
$$

so

$$
\text { variance }\left\{\sum_{1}^{n} x(t)\right\}=\int_{-.5}^{.5} \frac{\left|1-e^{2 \pi i u n}\right|^{2}}{\left|1-e^{2 \pi i u}\right|^{2}} d F(u)
$$

where $F(u)=\|x(0) E(u)\|^{2},-.5 \leqq u \leqq .5$, is the spectral distribution function. Hence we have

$$
\begin{aligned}
B>\text { variance }\left\{\sum_{1}^{n} x(t)\right\}= & \left\{\int_{-.5}^{0-}+\int_{0+}^{.5}\right\} \frac{\sin ^{2} \pi u n}{\sin ^{2} \pi u} d F(u) \\
& +n^{2}[F(0+)-F(0-)]
\end{aligned}
$$

which shows that $F(0+)-F(0-)$ must vanish. Moreover, we have

$$
\begin{aligned}
B & >\frac{1}{N} \sum_{n=1}^{N}\left\{\int_{-.5}^{0-}+\int_{0+}^{.5}\right\} \frac{\sin ^{2} \pi u n}{\sin ^{2} \pi u} d F(u) \\
& =\left\{\int_{-.5}^{0-}+\int_{0+}^{.5}\right\}\left[\frac{1}{N} \sum_{n=1}^{N}\left(\frac{1}{2}-\frac{1}{2} \cos 2 \pi u n\right)\right] \sin ^{-2} \pi u d F(u) .
\end{aligned}
$$

Clearly the limit of the expression in brackets, as $N \rightarrow \infty$, is $1 / 2$, so $\int_{-.5}^{.5} \sin ^{-2} \pi u d F(u)$ is finite. Q.E.D. (1).

The distribution function $F(u)$ defines a Lebesgue-Stieltjes measure on the real line segment $-.5 \leqq u \leqq .5$. Let $W$ denote the $L^{2}$ space of complex-valued measurable functions $\Phi(u)$ defined on $-.5 \leqq u \leqq .5$ for this measure. Define a correspondence between an element $x$ of $X$ and an element $\Phi(u)$ of $W$ by

$$
x=\int_{-.5}^{.5} \Phi(u) d x(0) E(u) \leftrightarrow \Phi(u)
$$

Then Stone [5] and Kolmogorov [1] have shown that this correspondence establishes an isomorphism between $X$ and $W$ that preserves inner products. The function $e^{2 \pi i u t} /\left(1-e^{2 \pi i u}\right)$ belongs to $W$ since

$$
\int_{-.5}^{.5}\left|\frac{e^{2 \pi i u t}}{1-e^{2 \pi i u}}\right|^{2} d F(u)=\frac{1}{4} \int_{-.5}^{.5} \sin ^{-2} \pi u d u<\infty .
$$


If we define the element $y(t)$ of $X$ by the correspondence $y(t)$ $\leftrightarrow e^{2 \pi i u t} /\left(1-e^{2 \pi i u}\right)$ we see that

$$
y(t)-y(t+1) \leftrightarrow \frac{e^{2 \pi i u t}-e^{2 \pi i u(t+1)}}{1-e^{2 \pi i u}}=e^{2 \pi i u t} .
$$

But by the spectral representation, we know that $x(t) \leftrightarrow e^{2 \pi i u t}$, and hence we have $y(t)-y(t+1)=x(t)$ for all integers $t$. Since

$$
\begin{aligned}
(y(t+s), y(t)) & =\int_{-.5}^{.5} \frac{e^{2 \pi i u(t+s)} e^{-2 \pi i u t}}{\left|1-e^{2 \pi i u}\right|^{2}} d F(u) \\
& =\frac{1}{4} \int_{-.5}^{.5} e^{2 \pi i u s} \sin ^{-2} \pi u d F(u)
\end{aligned}
$$

depends only on $s$, we see that $y(t)$ is a stationary random process. Q.E.D. (2).

Proof. (Sufficient conditions). Let condition (1) of the theorem be given. Since

$$
\text { variance }\left\{\sum_{1}^{n} x(t)\right\}=\int_{-.5}^{.5} \frac{\sin ^{2} \pi u n}{\sin ^{2} \pi u} d F(u) \leqq \int_{-.5}^{.5} \sin ^{-2} \pi u d F(u)
$$

we see that the variance is bounded. Q.E.D. (1).

Let condition (2) of the theorem be given. Then $\|y(t)\|$ is a finite constant. Because $\sum_{1}^{n} x(t)=y(1)-y(n+1)$ we have $\left\|\sum_{1}^{n} x(t)\right\|$ $\leqq\|y(1)\|+\|y(n+1)\|$, and so variance $\left\{\sum_{1}^{n} x(t)\right\}=\left\|\sum_{1}^{n} x(t)\right\|^{2}$ is bounded. Q.E.D. (2).

\section{REFERENCES}

1. A. N. Kolmogorov, Stationary sequences in Hilbert space, Bull. Moscow State Univ. Ser. Math. vol. 2 no. 6 (1941) 40 pp.

2. J. von Neumann, Eigenwerttheorie Hermitescher Functionaloperatoren, Math. Ann. vol. 102 (1929) pp. 49-131.

3. - Proof of the quasiergodic hypothesis, Proc. Nat. Acad. Sci. U.S.A. vol. 18 (1932) pp. 70-82.

4. A. Ja. Khintchine, Korrelationstheorie der stationären stochastischen Prozesse, Math. Ann. vol. 109 (1934) pp. 604-615.

5. M. H. Stone, Linear transformations in Hilbert space, Amer. Math. Soc. Colloquium Publications, vol. 15, 1932.

Mathematics Research Center, United States Army, MADISON, Wisconsin 